\title{
Scheduling Performance in Downlink WCDMA Networks with AMC and Fast Cell Selection
}

\author{
Hua Fu and Dong In Kim, Senior Member, IEEE
}

\begin{abstract}
This paper is concerned with the analysis of scheduling performance in downlink WCDMA networks that employ adaptive modulation and coding (AMC) and fast cell selection (FCS). The scheduling schemes investigated include (i) the Round Robin (RR) scheme, (ii) the maximum carrier-to-interference ratio $(C / I)$ scheme, (iii) the simplified proportional fair (PF) scheme, and (iv) the conventional PF scheme. The channel model includes large-scale signal attenuation and small-scale fading. By using a form analogous to Shannon's channel capacity formula, a logarithmic relationship between the instantaneous data rate and the $C / I$ with AMC is first established. Using the so-called Poisson scheme in which the probabilities of the occurrence of the events in Bernoulli trials depend on the trial index, FCS implementation is then examined in detail. Finally, a complete set of analytical expressions on the average system throughput, the peak data rate and the fairness for the abovementioned scheduling schemes are derived.
\end{abstract}

Index Terms-Throughput, fairness, scheduling, round robin, proportional fair, maximum $C / I$, AMC, FCS, WCDMA.

\section{INTRODUCTION}

$\mathbf{I}$ $\mathrm{N}$ parallel with fast growing internet services via the wireless medium, the third-generation $(3 \mathrm{G})$ mobile communication system based on wideband code division multiple access (WCDMA) has been developed as the basic radio access technology for the universal mobile telecommunications system (UMTS), and is being deployed as one of the IMT2000 standards [1] - [7]. The system can support high-speed packet data services with multi-rate multi-media traffic and with different quality-of-service (QoS) requirements.

In 3G WCDMA data networks, the downlink bears a significantly higher traffic load than the uplink [1]. In order to support this high traffic load, several new techniques are introduced, including downlink scheduling, adaptive modulation and coding (AMC) and fast cell selection (FCS). Downlink scheduling is the algorithm that decides which packets should be transmitted and when. In the WCDMA high-speed downlink packet access (WCDMA-HSDPA) network [2], [5], [7] and the CDMA high data rate (CDMA-HDR) network [3],

Manuscript received November 18, 2006; revised January 31, 2007, May 21, 2007, and July 29, 2007; accepted October 2, 2007. The associate editor coordinating the review of this paper and approving it for publication was S. Shen. This work was supported by the Ministry of Knowledge Economy, Korea, under the ITRC (Information Technology Research Center) support program supervised by the IITA (Institute of Information Technology Assessment). This paper was presented in part at the 2007 IEEE Global Communications Conference, Washington, USA, November 2007.

$\mathrm{H}$. Fu is with the Department of Electrical and Computer Engineering, National University of Singapore, Singapore 117576 (e-mail: elefh@nus.edu.sg).

D. I. Kim is with the School of Information and Communication Engineering, Sungkyunkwan University, Suwon 440-746, Korea (e-mail: dikim@ece.skku.ac.kr).

Digital Object Identifier 10.1109/TWC.2008.060956.
[4], [6], a feedback mechanism has been included to constantly estimate the channel and inform the base station (BS) of the channel states for all active mobile users. This information allows the BS some flexibility in scheduling according to the estimated channel quality. Several scheduling schemes have been proposed [1] - [7] to achieve the trade-off between throughput and fairness which are two key measures of the QoS. These scheduling schemes include the Round Robin (RR) [2], [5], [7], the maximum carrier-to-interference ratio $(C / I)$ [2], [5], [7], and the proportional fair (PF) [3], [4], [6]. Intuitively, the RR scheme is perhaps the simplest of all as its implementation completely ignores the channel conditions. The maximum $C / I$ scheme provides the maximum system throughput as it assigns the resource to the user with the best channel condition. The PF scheme schedules users to get access to the channel subject to some fairness criteria. AMC and FCS are two important link adaptation techniques. They have been proposed and implemented in 3G WCDMA downlink networks to enable more reliable communications while simultaneously improving data throughput and system capacity [6], [7]. Using AMC, the modulation and coding formats can be changed in accordance with variations in the channel conditions. For instance, the mobile users experiencing good channel quality can be assigned higher-order modulation and coding rate to achieve larger system throughput, whereas the users with poor channel quality may choose lower-order modulation and coding rate to combat channel impairments and enhance the performance. Using FCS, the mobile user can select the best active BS which should serve it on the downlink. It can be treated as alternative to soft handoff. Note that in current WCDMA-HSDPA and CDMA-HDR downlink systems, no soft handoff is used.

Various studies have been performed on AMC and FCS. In [8], the physical layer performance of the modems for high-order modulation schemes with AMC was extensively analyzed. A new method for selecting the appropriate AMC schemes in $3 \mathrm{G}$ wireless systems was proposed in [9]. The benefits of site selection diversity transmission power control for CDMA forward link were investigated in [10]. A network controlled cell selection for HSDPA and its advantage were studied in [11]. Some recent work on downlink scheduling included the following. A flexible downlink scheduling scheme used in packet data system was discussed in [12]. Downlink scheduling optimization with fairness constraint and user constraint was studied in [13] and [14], respectively. A crosslayer design on adaptive downlink scheduling is presented in [15]. The merit on base-station density in CDMA system using downlink scheduling was examined in [16]. However, 
the analysis on the impact of joint downlink scheduling and link adaptation on the throughput and fairness performance has not been addressed satisfactorily in the literature. The main contribution of this paper, therefore, is to develop a unified theoretical framework to determine the analytical expressions on the throughput and fairness, in which both downlink scheduling schemes and link adaptation techniques proposed in 3GPP and 3GPP2 [1] are incorporated. This work can be seen as a complement to the system level simulations and experimental implementations conducted in the industry [1] - [7]. Specifically, it derives a complete set of expressions on the BS throughput, the ensemble average throughput of the active region, the peak data throughput and the fairness with AMC, FCS and four scheduling algorithms, including the RR, maximum $C / I$, simplified $\mathrm{PF}$ and conventional PF. In the fairness analysis, two criteria are considered. From the expressions derived, the behavior of throughput and fairness as a function of various system parameters can easily be deduced. The results are not only of fundamental theoretical value, but are also vital to guiding the simulations and experiments to be performed.

Section II describes the system model. Section III presents AMC and establishes a logarithmic relationship between the instantaneous data rate and $C / I$. Section IV studies FCS using Possion scheme. In Section $\mathrm{V}$, the throughput and fairness of various scheduling schemes are analyzed, and their explicit expressions are derived. Section VI presents numerical/simulation results to highlight the dependence of throughput/fairness performance on FCS, scheduling algorithms and system parameters.

\section{System Model And Assumptions}

The network and system model consists of multiple hexagonal cells with a BS at its center, as shown in Fig. 1. Note that in theory the number of cells can be infinite, however, in actual simulation/numerical evaluation presented in Section VI, we only consider three concentric rings around the region studied. We assume $120^{\circ}$ ideal antenna sectorization, which is defined as unity gain inside sector, zero gain outside sector [17, Section (6.8)]. No power control is used and the total transmit powers are equal for all BSs. The active region modelled includes three contiguous sectors - the shaded region in Fig. 1. We assume that there are $M$ mobile users, uniformly distributed in the active region, and all BSs operate at full loading [4]. A set $S$ of BSs that provide the packet data service to the users in the active region includes BS 1, BS 2 and BS 3 only, i.e., $S=\{1,2,3\}$. In the downlink WCDMA system, the packet transmission can be carried out on a set of physical channels shared by the users with either code-division multiplexing (CDM) mode or time-division multiplexing (TDM) mode [1], [5]. In this paper we follow the transmission scheme used in the WCDMA-HSDPA and CDMA-HDR networks [3] - [7], and assume that the packets are transmitted in the TDM mode and at the full power allocated to a mobile user in an one-by-one transmission fashion with both data rate and time slot length changing [3, Figure 1], according to the user's channel conditions. At any given time the users receive the packet data from no more than one $\mathrm{BS}$ in the active set $S$, and we assume soft handoff is not utilized [6], [7]. Instead

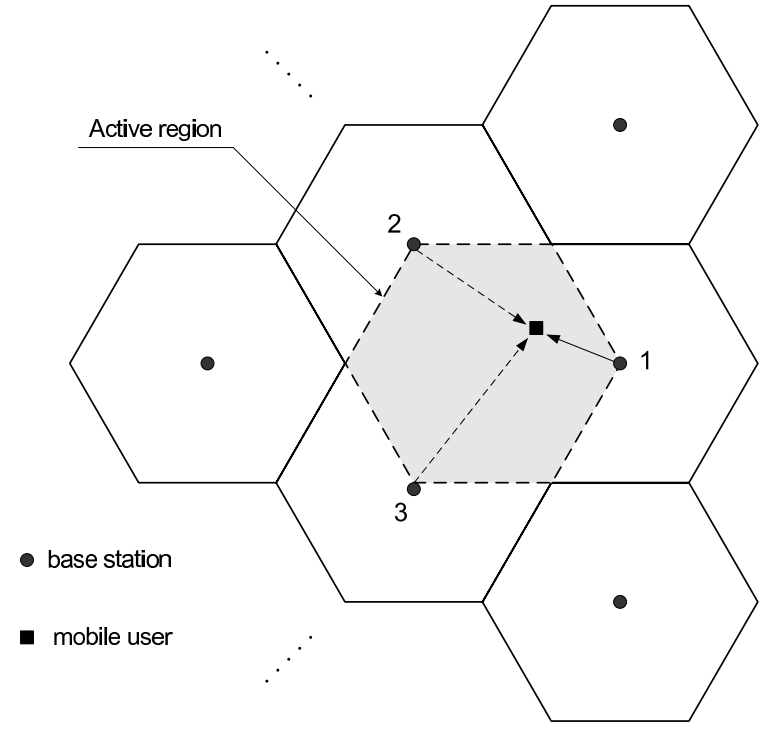

Fig. 1. Cellular layout and the active region for fast cell selection (FCS).

of combining transmit energies from multiple BSs, the users in the active region are now able to rapidly switch from communicating with one BS to another in the set $S$.

The channel model considered in this paper includes largescale signal attenuation and small-scale fading [17]. Largescale attenuation consists of path loss and shadowing effect which describe the mean signal variations over a much longer time scale and a large distance between the transmitter and receiver. The rapid fluctuations of the received signal envelope over a short period of time or travel distance are characterized by small-scale fading. We assume that the distance between the BS and mobile user is known. This is justifiable because the mobile users can periodically report their locations to the BS for handoff. The user location can also be detected by the network. UMTS has specified various kinds of mobile positioning methods [1], [18], [19], e.g., the cell coverage-based positioning method, the observed time difference of arrivalidle period downlink method, and the network-assisted GPS method. Therefore, the distance between the BS and mobile user can be accurately evaluated based on the knowledge of user's location. The small-scale channel fading in this paper is assumed to follow the Rayleigh distribution for the received signal envelope. The corresponding squared envelope (denoted as $\psi$ ) that is proportional to a short-term instantaneous power is exponentially distributed. Its probability density function (PDF) has the form [17]

$$
f(\psi)=\frac{1}{\eta^{2}} e^{-\psi / \eta^{2}}, \quad \psi \geq 0
$$

where $\eta^{2}$ is the average power. Following [17], [20] and [21], the signal power attenuation, $A(d, \xi, \psi)$, which includes path loss, lognormal shadowing and Rayleigh fading, is modelled as

$$
A(d, \xi, \psi)=d^{-4} 10^{(\xi / 10)} \psi
$$

where $d$ is the known distance between the BS and mobile user. The inverse fourth-law propagation is assumed. The quantity $\xi$ is a Gaussian-distributed random variable with mean zero and standard deviation $\sigma(\mathrm{dB})$, i.e., $\xi \sim N\left(0, \sigma^{2}\right)$, 
so that the shadowing $10^{(\xi / 10)}$ is a lognormally distributed random variable.

\section{CARRIER-TO-INTERFERENCE RATIO AND INSTANTANEOUS DATA RATE WITH AMC}

In the downlink WCDMA system, the user's channel condition measured by $C / I$ is a primary factor in determining the instantaneous data rate. The value of $C / I$ is defined as the ratio of the received signal energy density to single-sided interference density and can be measured through the pilot bursts [3]. Note that the interference here comprises intercell interference and thermal noise. It is assumed in this paper that the cell-specific spreading codes are statistically independent, so that the despread signals associated with BSs in the set $S$ can be treated as mutually independent under the TDM (i.e., no intracell interference). We use $W$ to denote the system bandwidth, $P$ to denote the total transmitted power of each BS, and $I_{k}+\nu$ to denote the mean interference plus background noise level received by user $k$ in the active region [22]. With TDM, the received instantaneous $C / I$ at user $k$ from $\mathrm{BS} i$ is written as

$$
\gamma_{k i}=\frac{P A\left(d_{k i}, \xi_{k i}, \psi_{k i}\right) / W}{\left(I_{k}+\nu\right) / W}=\frac{A\left(d_{k i}, \xi_{k i}, \psi_{k i}\right)}{\left(I_{k}+\nu\right) / P}
$$

where $i \in S=\{1,2,3\}$ and $k=1,2, \ldots, M$. Substituting (2) into (3) yields

$$
\gamma_{k i}=\frac{P d_{k i}^{-4}}{I_{k}+\nu} 10^{\left(\xi_{k i} / 10\right)} \psi_{k i}
$$

where, for each $k=1,2, \ldots, M$ and each $i=1,2,3,\left\{\xi_{k i}\right\}$ is a set of independent, identically distributed (i.i.d.) Gaussian random variables with mean zero and variance $\sigma^{2}(\mathrm{~dB}) ;\left\{\psi_{k i}\right\}$ is a set of i.i.d. exponential random variables with mean $\eta^{2}$. For each $k$ and $i$, the quantities $\xi_{k i}$ and $\psi_{k i}$ are mutually independent. For $k \neq p$ and $i \neq q,\left\{\xi_{k i}, \psi_{k i}\right\}$ are independent of $\left\{\xi_{p q}, \psi_{p q}\right\}$. The average value of $C / I$ is given by $\bar{\gamma}_{k i}=$ $\frac{P d_{k i}^{-4}}{I_{k}+\nu} \eta^{2} \exp \left[\frac{\sigma^{2}}{2}\left(\frac{\ln 10}{10}\right)^{2}\right]$.

The composite distribution due to shadowing $10^{\left(\xi_{k i} / 10\right)}$ and Rayleigh fading $\psi_{k i}$ in (4) called Lee-Yeh distribution [21] has no closed-form solution and is rather complicated mathematically, but can well be approximated by a lognormal function. This approximation is very useful and convenient to engineering applications since the lognormal distribution can be completely specified by the mean and standard deviation. Following [21] and using the natural logarithm, the PDF of $\gamma_{k i}$ can be derived as

$$
f\left(\gamma_{k i}\right)=\frac{1}{\gamma_{k i} \sqrt{2 \pi \varsigma^{2}}} \exp \left[-\frac{\left(\ln \gamma_{k i}-\mu_{k i}\right)^{2}}{2 \varsigma^{2}}\right]
$$

where the mean $\mu_{k i}$ and standard deviation $\varsigma$ are given, respectively, by

$$
\begin{aligned}
\mu_{k i} & =\ln \bar{\gamma}_{k i}-\left(\sigma^{2} / 2\right)(\ln 10 / 10)^{2}-2.5(\ln 10 / 10) \\
\varsigma^{2} & =\left(\sigma^{2}+5.57^{2}\right)(\ln 10 / 10)^{2} .
\end{aligned}
$$

The corresponding cumulative distribution function (CDF), denoted by $\Gamma\left(\gamma_{k i}\right)$, has the expression

$$
\Gamma\left(\gamma_{k i}\right)=1-\frac{1}{2} \operatorname{erfc}\left(\frac{\ln \gamma_{k i}-\mu_{k i}}{\varsigma \sqrt{2}}\right)=Q\left(\frac{\mu_{k i}-\ln \gamma_{k i}}{\varsigma}\right)
$$

where $\operatorname{erfc}(x)$ is the complementary error function and $Q(x)$ is the Gaussian $Q$-function. From (5) and (6), we see that the instantaneous $C / I\left\{\gamma_{k i}\right\}$ is a set of independent, nonidentically distributed lognormal random variables. The means $\left\{\mu_{k i}\right\}$ depend on the parameter $\eta^{2}$ of fast Rayleigh fading, the transmitted signal power $P$, the interference level $I_{k}+\nu$ and the mobile user location in the active region, while the variances $\left\{\varsigma^{2}\right\}$ depend on the standard deviation $\sigma$ of the lognormal shadow fading.

The AMC technique is a fundamental feature in the WCDMA-HSDPA and CDMA-HDR networks. It allows to optimize the modulation and coding formats adaptive to variations in the channel conditions. Since each value of the user's measured $C / I$ can be mapped directly into an instantaneous data rate that such value can support for a given target error performance, the downlink WCDMA system can support dynamic packet data rates through AMC. For example, in the CDMA-HDR system, for BS $i$ and user $k$, the relationship between the instantaneous data rate $R_{k i}$ and the $C / I \gamma_{k i}$ for different modulation orders and coding rates is determined by using a look-up mapping table [3, Table 2]. Mathematically, the linear relationship between the instantaneous data rate $R_{k i}$ and the $C / I \gamma_{k i}$ can be formulated as [17], [23], [24]

$$
R_{k i}=\frac{W}{\left(E_{b} / I_{0}\right)_{k}} \gamma_{k i}
$$

where $\left(E_{b} / I_{0}\right)_{k}$ is the required bit energy-to-interferenceplus-noise density ratio, whose value depends on modulation and coding formats, channel impairments such as fading, and error performance requirements. For instance, in [3, Table 2] and [6, Table 1], with iterative decoding on serial concatenated decoding and QPSK modulation, a 1-percent packet-error rate (PER) corresponds to $\left(E_{b} / I_{0}\right)_{k} \approx 2.5 \mathrm{~dB}$ at the lower data rates, ranging from $38.4 \mathrm{~kb} / \mathrm{s}$ to $1228.8 \mathrm{~kb} / \mathrm{s}$. This implies that the supportable data rate is linearly proportional to $C / I$ over the wide region of lower data rates, which can be well approximated by (8). However, at the region of higher data rates such as $1843.2 \mathrm{~kb} / \mathrm{s}$ and $2457.6 \mathrm{~kb} / \mathrm{s}$ in [3, Table 2], in order to achieve the 1-percent PER, the required $\left(E_{b} / I_{0}\right)_{k}$ will increase considerably because higher-order modulation (e.g., 8PSK and 16QAM) is more sensitive to fading and interference. Therefore, in the case of AMC, the linear relationship (8) is not convenient to use, since we have to keep adjusting the quantity $\left(E_{b} / I_{0}\right)_{k}$ based on the modulation and coding formats. It is more desirable to find a simple mathematical function that can relate $R_{k i}$ directly to $\gamma_{k i}$. This can be done by using Shannon's channel capacity formula where a logarithmic relationship is employed. We proceed first with using the logarithmic relation to approximate (8) with $\left(E_{b} / I_{0}\right)_{k} \approx 2.5 \mathrm{~dB}$ for a small value of $C / I$ at the lower data rates.

At low signal-to-noise ratio (SNR), Shannon's channel capacity formula is approximated to [25]

$$
\frac{C}{W}=\log _{2}(1+\mathrm{SNR})=\frac{\ln (1+\mathrm{SNR})}{\ln 2} \approx \frac{\mathrm{SNR}}{\ln 2}
$$

where the following relation has been used for low SNR

$$
\ln (1+\mathrm{SNR})=\mathrm{SNR}-\frac{1}{2}(\mathrm{SNR})^{2}+\frac{1}{3}(\mathrm{SNR})^{3} \cdots
$$




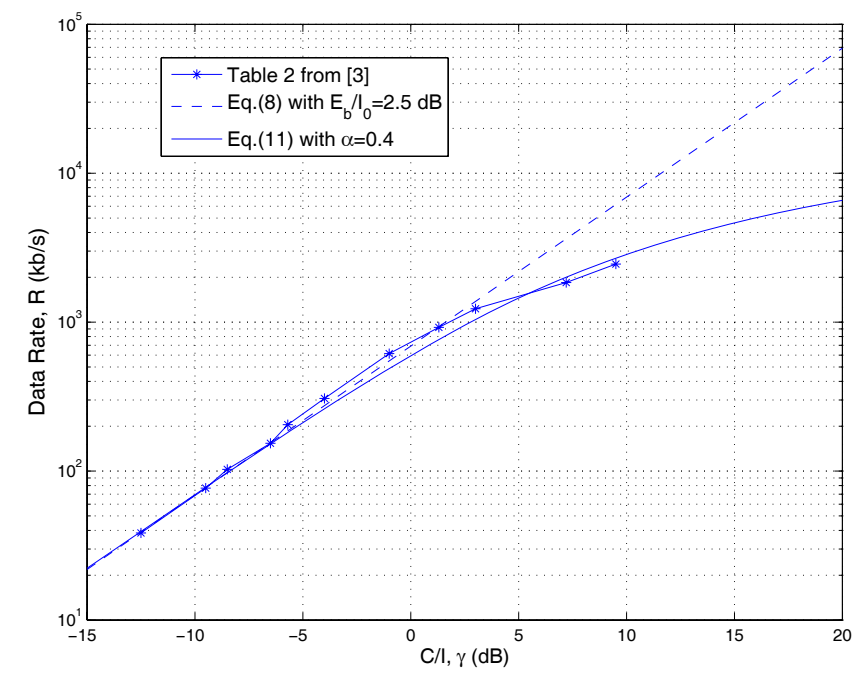

Fig. 2. Linear and logarithmic relationships between the instantaneous data rate and the $C / I$.

Now we can approximate (8) by a function in a form analogous to (9), by defining

$$
\alpha=\frac{\ln 2}{\left(E_{b} / I_{0}\right)_{k}}=\frac{\ln 2}{10^{2.5 / 10}}=0.4 .
$$

Using this and (9), we derive the following formula

$$
R_{k i}=\frac{W}{\left(E_{b} / I_{0}\right)_{k}} \gamma_{k i}=W \frac{\alpha \gamma_{k i}}{\ln 2} \approx W \log _{2}\left(1+\alpha \gamma_{k i}\right)
$$

which gives a logarithmic relationship between the data rate $R_{k i}$ and the $C / I$. To show the validity of (11), the data rate $R_{k i}$ is plotted against the $C / I, \gamma_{k i}$, in Fig. 2, by using (8) and (11) with system bandwidth $W=1.2288 \mathrm{MHz}$. As a basis for comparison, the look-up mapping table given in [3, Table 2] is also plotted. From Fig. 2, we see that for low $C / I$ at the region of lower rates, the mapping table [3, Table 2] can reasonably be approximated by both linear relationship (8) and logarithmic relationship (11). However, in order for (8) to be valid as $C / I$ increases, we need to modify $\left(E_{b} / I_{0}\right)_{k}$ to a larger value accordingly. In other words, the linear relationship (8) can only provide good approximation between the instantaneous data rate and the $C / I$ locally, whereas the logarithmic relationship (11) is more convenient to use and can be utilized for the reasonably accurate analysis globally.

\section{Fast Cell Selection and Downlink Scheduling}

FCS has been proposed for both WCDMA-HSDPA network and CDMA-HDR network. Using FCS, each mobile user in the active region can request data transmission from three BS candidates of the active set $S=\{1,2,3\}$, but only selects the best BS which should serve it at any time. FCS can potentially increase the $C / I$, and thus, increase data transmission rate. Therefore, FCS has become a key element of downlink WCDMA networks that determines an overall system performance such as throughput [6], [7]. In practice, the user can continuously monitor all three BSs in the set $S$ and report to the radio network controller (RNC) when a change of the best serving BS occurs. Determination of the best BS may be based on the user's $C / I$ measurements. In general, FCS can be performed in the following procedure:

1) Measurements on each mobile user's $C / I$ from the three BSs in the set $S$ are made,

2) Based on the measurements of $C / I$, each mobile user in the active region selects the best serving BS,

3) Each mobile user then transmits a BS indicator (as to the best serving BS) along with its measurement on $C / I$ using the uplink dedicated control channel,

4) Each BS in the set $S$ uses the $C / I$ measurements to determine scheduling priorities as well as the AMC level for a scheduled user via the scheduling algorithm used.

From the above procedure, we note that FCS is carried out for every user in the active region before downlink scheduling is implemented. After FCS each user is assigned to one and only one of the possible BSs $S=\{1,2,3\}$. Therefore, we can visualize the FCS procedure as a partitioning of the whole users in the active region into disjoint groups, say $\left\{G_{i}\right\}, i=$ $1,2,3$. Each group $G_{i}$ comprises all users such that the BS $i$ is the best serving BS. Once the groups have been formed, a scheduling scheme will be employed to each group $G_{i}$ to decide which user in the group the data should be transmitted to. In the following, we will apply Poisson scheme [26] to study FCS.

Without loss of generality, we can choose BS 1 as the best serving BS and form the group $G_{1}$. The decision process given in steps 1) - 3) above can be modelled as a generalized Bernoulli trial, called the Possion scheme. This process is similar to the coin tossing problem. Specifically, the FCS decision for each of $M$ mobile users can be viewed as performing $M$ random trials to decide which user in the active region will be served by BS 1 (i.e., in the group $G_{1}$ ). Clearly, the outcomes of the $M$ trials are independent. As a result of the $k$ th trial $(k=1,2, \ldots, M)$, we can obtain the event $A_{k 1}$, which indicates that user $k$ is in the group $G_{1}$, with probability $p_{k 1}$, or the complementary $\bar{A}_{k 1}$, which indicates that user $k$ is not served by BS 1 , with probability $1-p_{k 1}$. Since the selection criterion for determining the best serving BS is based on the user's $C / I$, the event $A_{k 1}$ occurs if both the $C / I$ 's $\gamma_{k 2}$ and $\gamma_{k 3}$ are simultaneously below $\gamma_{k 1}$. Hence, the probability $p_{k 1}$ is written as

$$
p_{k 1}=\operatorname{Pr}\left[\gamma_{k 2} \leq \gamma_{k 1}, \gamma_{k 3} \leq \gamma_{k 1}\right] .
$$

We can evaluate the probability $p_{k 1}$ in (12) by first conditioning on knowing $\gamma_{k 1}$. By averaging this conditional probability over the distribution of $\gamma_{k 1}$, the final probability $p_{k 1}$ is obtained. Since $\left\{\gamma_{k i}\right\}_{i=1}^{3}$ are mutually independent random variables, the conditional probability is the product of the probabilities that the quantities $\gamma_{k 2}$ and $\gamma_{k 3}$ are smaller than $\gamma_{k 1}$. Using (6) and (7) in (12), the final result is derived as

$$
\begin{gathered}
p_{k 1}=\frac{1}{\sqrt{2 \pi \varsigma^{2}}} \int_{0}^{\infty} \frac{1}{x} Q\left(\frac{\mu_{k 2}-\ln x}{\varsigma}\right) Q\left(\frac{\mu_{k 3}-\ln x}{\varsigma}\right) \\
\quad \times \exp \left[-\frac{\left(\ln x-\mu_{k 1}\right)^{2}}{2 \varsigma^{2}}\right] d x
\end{gathered}
$$

Note that unlike the coin-tossing Bernouli trial, the probabilities $\left\{p_{k 1}\right\}$ of occurrence of the events $\left\{A_{k 1}\right\}_{k=1}^{M}$ in the generalized Bernoulli trials are distinct from one another. 
There are two possible events ( $A_{k 1}$ and the complementary $\bar{A}_{k 1}$ ) belonging to each user $k$, and $M$ users in the active region; it follows that there are $2^{M}$ distinct ways of assigning the $M$ users to $G_{1}$, each of which stands for one of the possible sequence of length $M$ constructible from $A_{k 1}$ and the complementary $\bar{A}_{k 1}$. If we associate with every possible sequence a probability $P_{l}$ for $l=1,2, \ldots, 2^{M}$, we have

$$
\sum_{l=1}^{2^{M}} P_{l}=1 .
$$

As an example, suppose there are three users in the active region (i.e., $M=3$ ), we have $2^{3}=8$ distinct ways to assign the three users to $G_{1}$. Table 1 gives all events and the corresponding probabilities of $G_{1}$. Clearly, in the case of $l=1$, where $P_{1}=\left(1-p_{11}\right)\left(1-p_{21}\right)\left(1-p_{31}\right)$, no user is served by BS 1 , while in the case of $l=8$, where $P_{8}=p_{11} p_{21} p_{31}$, all three users are served by BS 1 .

Without loss of generality and for the purpose of notational simplicity, in the following analysis, we assume that after FCS, the users from 1 to $K$, where $K<M$, are in the group $G_{1}$, and the rest of $M-K$ users are served by either BS 2 or BS 3 . The probability of this assignment, denoted by $P$, is thus given by

$$
P=\prod_{k=1}^{K} p_{k 1} \prod_{k=K+1}^{M}\left(1-p_{k 1}\right) .
$$

Now, we have $K$ users $(k=1,2, \ldots, K)$ in $G_{1}$. The scheduling scheme in the abovementioned step 4) will then decide which user in the group $G_{1}$ is served by BS 1 with its requested data rate at any given time. The scheduling scheme is another key element that determines an overall system performance for downlink WCDMA networks. A detailed discussion on downlink scheduling is available in [2] - [7], [23], [24]. In the next section, we will study four downlink scheduling schemes, namely, the RR, maximum $C / I$, simplified $\mathrm{PF}$ and conventional PF, and derive their throughput and fairness expressions for BS 1 with partitioning group $G_{1}$ and corresponding probability assignment $P$.

\section{Throughrut and Fairness Performance}

We note that after FCS, the events $\left\{A_{k 1}\right\}_{k=1}^{K}$ occur. Thus, given $A_{k 1}(k=1,2, \ldots, K)$, the density function of $\gamma_{k 1}$ in group $G_{1}$ has changed from $f\left(\gamma_{k 1}\right)$ given by (5) to the following conditional pdf

$$
f_{c}\left(\gamma_{k 1}\right)=f\left(\gamma_{k 1} \mid A_{k 1}\right)=f\left(\gamma_{k 1} \mid \gamma_{k 2} \leq \gamma_{k 1}, \gamma_{k 3} \leq \gamma_{k 1}\right) .
$$

Using the mixed form of Bayes rule [27, eq. (2.103a)] in (16), we have

$$
f_{c}\left(\gamma_{k 1}\right)=\frac{\operatorname{Pr}\left[\gamma_{k 2} \leq \gamma_{k 1}, \gamma_{k 3} \leq \gamma_{k 1} \mid \gamma_{k 1}\right]}{\operatorname{Pr}\left[\gamma_{k 2} \leq \gamma_{k 1}, \gamma_{k 3} \leq \gamma_{k 1}\right]} f\left(\gamma_{k 1}\right)
$$

which leads to, after manipulation and simplification

$$
\begin{aligned}
f_{c}\left(\gamma_{k 1}\right)=\frac{\beta_{k 1}}{\gamma_{k 1}} & Q\left(\frac{\mu_{k 2}-\ln \gamma_{k 1}}{\varsigma}\right) Q\left(\frac{\mu_{k 3}-\ln \gamma_{k 1}}{\varsigma}\right) \\
& \times \exp \left[-\frac{\left(\ln \gamma_{k 1}-\mu_{k 1}\right)^{2}}{2 \varsigma^{2}}\right]
\end{aligned}
$$

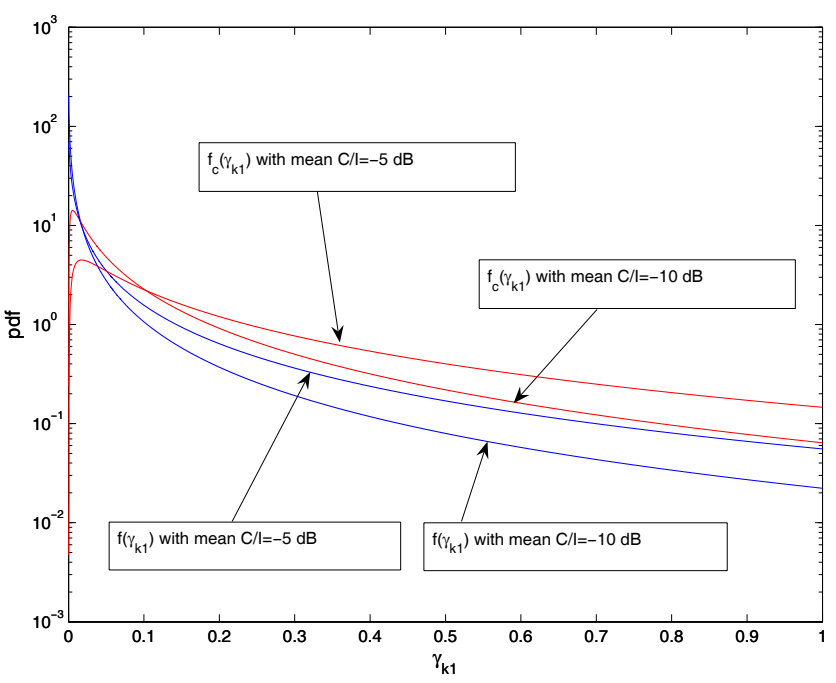

Fig. 3. The probability density functions (pdf's) of $C / I$ with and without FCS.

where $\beta_{k 1}$ is a constant quantity whose inverse value is given by

$$
\begin{aligned}
1 / \beta_{k 1}=\int_{0}^{\infty} & \frac{1}{x} Q\left(\frac{\mu_{k 2}-\ln x}{\varsigma}\right) Q\left(\frac{\mu_{k 3}-\ln x}{\varsigma}\right) \\
& \times \exp \left[-\frac{\left(\ln x-\mu_{k 1}\right)^{2}}{2 \varsigma^{2}}\right] d x .
\end{aligned}
$$

In Fig. 3, we plot the pdf's $f\left(\gamma_{k 1}\right)$ and $f_{c}\left(\gamma_{k 1}\right)$ when a user is at the center of the active region for $\eta^{2}=1$ and $\sigma=8$ (dB), with the mean $C / I$ as the parameter. We can see that for a fixed mean $C / I$, FCS can help increase the probability $\operatorname{Pr}\left[\gamma_{k 1}>\gamma_{0}\right]$, where $\gamma_{0}>0$ is some critical value. This implies that FCS can bring a statistical reduction of fading dip for the instantaneous $C / I$.

Following [3], the throughput is defined as the average data rate which is equal to the number of data bits divided by the total transmission time. We use $R_{k 1}$ bits/s $(k=1,2, \ldots, K)$ to denote the instantaneous data rate for user $k$ in $G_{1}$ to receive data from BS 1, and $S_{k 1}$ to denote the corresponding system access probability. The throughput is then expressed by

$$
\Lambda=\sum_{k=1}^{K} R_{k 1} S_{k 1} \quad \text { bits/s. }
$$

Note that, in (19) the quantities $R_{k 1}$ and $S_{k 1}$ are deterministic variables. If they are random variables, the throughput expression should be defined by $\Lambda=\mathbf{E}\left[\sum_{k=1}^{K} R_{k 1} S_{k 1}\right]$, where $\mathbf{E}$ denotes the expectation.

The fairness metric can be defined in two forms. The first form is defined in terms of the average data rate achieved by each user, which is given by [28], [29, eq. (33)]

$$
F=\frac{\left(\sum_{k=1}^{K} \mathbf{E}\left[R_{k 1} S_{k 1}\right]\right)^{2}}{K \sum_{k=1}^{K}\left(\mathbf{E}\left[R_{k 1} S_{k 1}\right]\right)^{2}}=\frac{\Lambda^{2}}{K \sum_{k=1}^{K}\left(\mathbf{E}\left[R_{k 1} S_{k 1}\right]\right)_{(20)}^{2}} .
$$

This definition has been used in the CDMA-HDR system [4], [6] to evaluate the PF scheme. The second definition of the 
TABLE I

EVENT AND PROBABILITY ASSIGNMENT IN $G_{1}$ FOR $M=3$

\begin{tabular}{|c|c|c|c|}
\hline Index $l$ & Event & Probability $P_{l}$ & $P_{l}$ value for scenario 6 \\
\hline 1 & $\bar{A}_{11} \bar{A}_{21} \bar{A}_{31}$ & $\left(1-p_{11}\right)\left(1-p_{21}\right)\left(1-p_{31}\right)$ & 0.2964 \\
\hline 2 & $A_{11} \bar{A}_{21} \bar{A}_{31}$ & $p_{11}\left(1-p_{21}\right)\left(1-p_{31}\right)$ & 0.1481 \\
\hline 3 & $\bar{A}_{11} A_{21} \bar{A}_{31}$ & $\left(1-p_{11}\right) p_{21}\left(1-p_{31}\right)$ & 0.1481 \\
\hline 4 & $\bar{A}_{11} \bar{A}_{21} A_{31}$ & $\left(1-p_{11}\right)\left(1-p_{21}\right) p_{31}$ & 0.1481 \\
\hline 5 & $A_{11} A_{21} \bar{A}_{31}$ & $p_{11} p_{21}\left(1-p_{31}\right)$ & 0.0741 \\
\hline 6 & $\bar{A}_{11} A_{21} A_{31}$ & $\left(1-p_{11}\right) p_{21} p_{31}$ & 0.0741 \\
\hline 7 & $A_{11} \bar{A}_{21} A_{31}$ & $p_{11}\left(1-p_{21}\right) p_{31}$ & 0.0741 \\
\hline 8 & $A_{11} A_{21} A_{31}$ & $p_{11} p_{21} p_{31}$ & 0.0370 \\
\hline
\end{tabular}

fairness metric is based on the system access probability for each user, which is [24]

$$
F^{\prime}=\frac{\left(\sum_{k=1}^{K} S_{k 1}\right)^{2}}{K \sum_{k=1}^{K} S_{k 1}^{2}}=\left(K \sum_{k=1}^{K} S_{k 1}^{2}\right)^{-1} .
$$

This definition has been used in the WCDMA-HSDPA system [5], [7] to initially study the RR and maximum $C / I$ schemes. In this paper we will evaluate both $F$ and $F^{\prime}$. However, we should stress that a fair scheduling scheme in terms of $F$ may become unfair if $F^{\prime}$ is used, and vice versa. The values of $F$ and $F^{\prime}$ range from $1 / K$ to 1 . The value of 1 corresponds to a totally fair allocation (with equal average data rate or access probability for all users). A totally unfair allocation (with full average data rate or chance of system access given to only one user) has a fairness of $1 / K$.

\section{A. Throughput and fairness of Round Robin scheme}

In the RR scheme, at any scheduling instant, the users are serviced in a round-robin fashion, i.e., the $K$ data slots are assigned one at a time successively for each user, and all users must be serviced before reservicing a user. Thus, the system access probability is $1 / K$ for all users (i.e., $S_{k 1}=1 / K$ ). The throughput can be expressed by

$$
\Lambda_{R R}=\mathbf{E}\left[\sum_{k=1}^{K} \frac{1}{K} R_{k 1}\right]=\frac{1}{K} \sum_{k=1}^{K} \int_{0}^{\infty} R_{k 1} f_{c}\left(\gamma_{k 1}\right) d \gamma_{k 1}
$$

where $R_{k 1}$ and $f_{c}\left(\gamma_{k 1}\right)$ are from (11) and (18), respectively. Substituting (11) and (18) into (22) yields

$$
\begin{aligned}
\Lambda_{R R}=\frac{1}{K} & \sum_{k=1}^{K} \int_{0}^{\infty} \frac{W \beta_{k 1} \log _{2}(1+\alpha x)}{x} Q\left(\frac{\mu_{k 2}-\ln x}{\varsigma}\right) \\
& \times Q\left(\frac{\mu_{k 3}-\ln x}{\varsigma}\right) \exp \left[-\frac{\left(\ln x-\mu_{k 1}\right)^{2}}{2 \varsigma^{2}}\right] d x
\end{aligned}
$$

which can be computed numerically. The fairness $F$ in terms of the average data rate can be evaluated as (24) shown at the top of next page. Clearly, in terms of the system access probability, we have $F_{R R}^{\prime}=1$, which states that the RR scheme is a totally fair allocation scheme.

\section{B. Throughput and fairness of maximum $C / I$ scheme}

In the maximum $C / I$ scheme, at any scheduling instant, all users are ranked based on their $C / I$ and then the packet data is transmitted to the user that experiences the largest $C / I$. Conditioning on $\gamma_{k 1}$, the access probability to BS 1 for user $k$ would be the probability that each of $\gamma_{j 1}(j=1,2, \ldots, K$ and $j \neq k$ ) is simultaneously smaller than $\gamma_{k 1}$. Since $\left\{\gamma_{j 1}\right\}$ is a set of independent random variables, this access probability will be the product of the independent probabilities that each $\gamma_{j 1}<\gamma_{k 1}$ for $j \neq k$. Thus, the throughput for the maximum $C / I$ scheme can be evaluated as

$$
\begin{aligned}
\Lambda_{C / I}= & \mathbf{E}\left\{\sum_{k=1}^{K} R_{k 1} \operatorname{Pr}\left[\max _{j \neq k}\left\{\gamma_{j 1}\right\} \leq \gamma_{k 1}\right]\right\} \\
= & \sum_{k=1}^{K} \int_{0}^{\infty} R_{k 1} \operatorname{Pr}\left[\max _{j \neq k}\left\{\gamma_{j 1}\right\} \leq \gamma_{k 1}\right] f_{c}\left(\gamma_{k 1}\right) d \gamma_{k 1} \\
= & \sum_{k=1}^{K} \int_{0}^{\infty} \frac{W \beta_{k 1} \log _{2}(1+\alpha x)}{x} Q\left(\frac{\mu_{k 2}-\ln x}{\varsigma}\right) \\
& \times Q\left(\frac{\mu_{k 3}-\ln x}{\varsigma}\right) \exp \left[-\frac{\left(\ln x-\mu_{k 1}\right)^{2}}{2 \varsigma^{2}}\right] \\
& \times\left\{\prod_{\substack{j=1 \\
j \neq k}}^{K} \int_{0}^{x} \frac{\beta_{j 1}}{\gamma_{j 1}} Q\left(\frac{\mu_{j 2}-\ln \gamma_{j 1}}{\varsigma}\right) Q\left(\frac{\mu_{j 3}-\ln \gamma_{j 1}}{\varsigma}\right)\right. \\
& \left.\times \exp \left[-\frac{\left(\ln \gamma_{j 1}-\mu_{j 1}\right)^{2}}{2 \varsigma^{2}}\right] d \gamma_{j 1}\right\} d x
\end{aligned}
$$

which can be computed numerically.

Since the system access probability for user $k$ is given by

$S_{k 1}^{C / I}=\int_{0}^{\infty} \operatorname{Pr}\left[\max _{j \neq k}\left\{\gamma_{j 1}\right\} \leq \gamma_{k 1}\right] f_{c}\left(\gamma_{k 1}\right) d \gamma_{k 1}=$

$\int_{0}^{\infty} \frac{\beta_{k 1}}{x} Q\left(\frac{\mu_{k 2}-\ln x}{\varsigma}\right) Q\left(\frac{\mu_{k 3}-\ln x}{\varsigma}\right) \exp \left[\frac{-\left(\ln x-\mu_{k 1}\right)^{2}}{2 \varsigma^{2}}\right]$

$\times\left\{\prod_{\substack{j=1 \\ j \neq k}}^{K} \int_{0}^{x} \frac{\beta_{j 1}}{\gamma_{j 1}} Q\left(\frac{\mu_{j 2}-\ln \gamma_{j 1}}{\varsigma}\right) Q\left(\frac{\mu_{j 3}-\ln \gamma_{j 1}}{\varsigma}\right)\right.$

$\left.\times \exp \left[-\frac{\left(\ln \gamma_{j 1}-\mu_{j 1}\right)^{2}}{2 \varsigma^{2}}\right] d \gamma_{j 1}\right\} d x$,

the fairness metric $F_{C / I}^{\prime}$ is evaluated as

$$
F_{C / I}^{\prime}=\left[K \sum_{k=1}^{K}\left(S_{k 1}^{C / I}\right)^{2}\right]^{-1} .
$$

In terms of the average data rate, the fairness metric $F_{C / I}$ is expressed by (28) shown at the top of next page. As the served users are the ones with the best channels in the maximum $C / I$ 


$$
F_{R R}=\frac{\Lambda_{R R}^{2} / K}{\sum_{k=1}^{K}\left\{\int_{0}^{\infty} \frac{W \beta_{k 1} \log _{2}(1+\alpha x)}{x} Q\left(\frac{\mu_{k 2}-\ln x}{\varsigma}\right) Q\left(\frac{\mu_{k 3}-\ln x}{\varsigma}\right) \exp \left[-\frac{\left(\ln x-\mu_{k 1}\right)^{2}}{2 \varsigma^{2}}\right] d x\right\}^{2}}
$$

$$
F_{C / I}=\frac{\Lambda_{C / I}^{2} / K}{\sum_{k=1}^{K}\left\{\int_{0}^{\infty} W \log _{2}\left(1+\alpha \gamma_{k 1}\right) \operatorname{Pr}\left[\max _{j \neq k}\left\{\gamma_{j 1}\right\} \leq \gamma_{k 1}\right] f_{c}\left(\gamma_{k 1}\right) d \gamma_{k 1}\right\}^{2}}
$$

scheme, we expect that $\Lambda_{C / I}$ will yield the highest throughput, whereas the values of $F_{C / I}^{\prime}$ and $F_{C / I}$ will decrease as the number of users $K$ increases since the users with poor channel conditions have little chance to access the system.

\section{Throughput and fairness of proportional fair scheme}

The PF scheme provides a compromise between the RR and maximum $C / I$ schemes. Thus, we expect that it can offer a good trade-off between the throughput and fairness. The $\mathrm{PF}$ scheduler has already been proposed and implemented for scheduling in real networks, such us Qualcomm's 1xEVolution system [6]. The basic feature of this scheduler is that in each transmission slot the packet data is only transmitted to the user that experiences the largest ratio of the instantaneous achievable data rate to the average served data rate. In other words, the criterion for scheduling a user is based on the user's relative data rate. Note that if the linear relationship (8) is used, the selection criterion based on the relative data rate will be equivalent to that based on the relative $C / I$, defined as the instantaneous- $C / I$-to-mean- $C / I$ ratio. This is because in the case of the linear relationship (8), we have the relation

$$
\frac{R_{k 1}}{\mathbf{E}\left[R_{k 1}\right]}=\frac{\left[\frac{W}{\left(E_{b} / I_{0}\right)_{k 1}} \gamma_{k 1}\right]}{\mathbf{E}\left[\frac{W}{\left(E_{b} / I_{0}\right)_{k 1}} \gamma_{k 1}\right]}=\frac{\gamma_{k 1}}{\mathbf{E}\left[\gamma_{k 1}\right]} .
$$

However, if the logarithmic relationship (11) is used, the relative data rate criterion differs from the relative $C / I$ criterion. In other words, the relative $C / I$ criterion is less valid when higher-order modulation and coding are used. A simplified version of the PF scheme is proposed in [23], where the user with the highest priority in a transmission slot is determined by the ratio of the instantaneous $C / I$ to the average data rate. We define the instantaneous- $C / I$-to-mean-data-rate ratio $\delta_{k 1}$ as

$$
\delta_{k 1}=\frac{\gamma_{k 1}}{\bar{R}_{k 1}}
$$

where $\bar{R}_{k 1}$ denotes the average data rate.

We first derive the throughput and fairness expressions for the simplified PF scheme. The procedure is parallel to that followed in the case of the maximum $C / I$ scheme. Using (11) and (18), the average data rate $\bar{R}_{k 1}$ can be evaluated as

$$
\begin{aligned}
\bar{R}_{k 1}=\int_{0}^{\infty} & \frac{W \beta_{k 1} \log _{2}(1+\alpha x)}{x} Q\left(\frac{\mu_{k 2}-\ln x}{\varsigma}\right) \\
& \times Q\left(\frac{\mu_{k 3}-\ln x}{\varsigma}\right) \exp \left[-\frac{\left(\ln x-\mu_{k 1}\right)^{2}}{2 \varsigma^{2}}\right] d x .
\end{aligned}
$$

Since $\delta_{k 1}$ is a linear transformation of $\gamma_{k 1}$ by the constant $1 / \bar{R}_{k 1}$, the PDF of $\delta_{k 1}$ can be derived as [30]

$$
\begin{aligned}
f_{c}\left(\delta_{k 1}\right)=\frac{\beta_{k 1}}{\delta_{k 1}} Q & \left(\frac{\mu_{k 2}-\ln \delta_{k 1}-\ln \bar{R}_{k 1}}{\varsigma}\right) \\
\times & Q\left(\frac{\mu_{k 3}-\ln \delta_{k 1}-\ln \bar{R}_{k 1}}{\varsigma}\right) \\
& \times \exp \left[-\frac{\left(\ln \delta_{k 1}+\ln \bar{R}_{k 1}-\mu_{k 1}\right)^{2}}{2 \varsigma^{2}}\right] .
\end{aligned}
$$

We note that $\left\{\delta_{k 1}\right\}_{k=1}^{K}$ is a set of independent random variables. Hence, conditioning on $\delta_{k 1}$, the system access probability of user $k$ is the product of the independent probabilities that each $\delta_{j 1}<\delta_{k 1}$ for $j \neq k$. The instantaneous data rate $R_{k 1}$ is now given by $R_{k 1}=W \log _{2}\left(1+\alpha \gamma_{k 1}\right)=W \log _{2}\left(1+\alpha \bar{R}_{k 1} \delta_{k 1}\right)$. Thus, the throughput for the simplified PF scheme can be evaluated

$$
\begin{aligned}
& \Lambda_{S P F}=\mathbf{E}\left\{\sum_{k=1}^{K} R_{k 1} \operatorname{Pr}\left[\max _{j \neq k}\left\{\delta_{j 1}\right\} \leq \delta_{k 1}\right]\right\} \\
& =\sum_{k=1}^{K} \int_{0}^{\infty} R_{k 1} \operatorname{Pr}\left[\max _{j \neq k}\left\{\delta_{j 1}\right\} \leq \delta_{k 1}\right] f_{c}\left(\delta_{k 1}\right) d \delta_{k 1}=W \\
& \times \sum_{k=1}^{K} \int_{0}^{\infty} Q\left(\frac{\mu_{k 2}-\ln x-\ln \bar{R}_{k 1}}{\varsigma}\right) Q\left(\frac{\mu_{k 3}-\ln x-\ln \bar{R}_{k 1}}{\varsigma}\right) \\
& \times \exp \left[-\frac{\left(\ln x+\ln \bar{R}_{k 1}-\mu_{k 1}\right)^{2}}{2 \varsigma^{2}}\right] \frac{\beta_{k 1} \log _{2}\left(1+\alpha \bar{R}_{k 1} x\right)}{x} \\
& \times\left\{\prod_{\substack{j=1 \\
j \neq k}}^{K} \int_{0}^{x} \frac{\beta_{j 1}}{\delta_{j 1}} Q\left(\frac{\mu_{j 2}-\ln \delta_{j 1}-\ln \bar{R}_{j 1}}{\varsigma}\right)\right. \\
& \quad \times Q\left(\frac{\mu_{j 3}-\ln \delta_{j 1}-\ln \bar{R}_{j 1}}{\varsigma}\right) \\
& \left.\quad \times \exp \left[-\frac{\left(\ln \delta_{j 1}+\ln \bar{R}_{j 1}-\mu_{j 1}\right)^{2}}{2 \varsigma^{2}}\right] d \delta_{j 1}\right\} d x .
\end{aligned}
$$

Since the system access probability of user $k$ is now given 
by

$$
\begin{aligned}
& S_{k 1}^{S P F}=\int_{0}^{\infty} \operatorname{Pr}\left[\max _{j \neq k}\left\{\delta_{j 1}\right\} \leq \delta_{k 1}\right] f_{c}\left(\delta_{k 1}\right) d \delta_{k 1} \\
& =\int_{0}^{\infty} Q\left(\frac{\mu_{k 2}-\ln x-\ln \bar{R}_{k 1}}{\varsigma}\right) Q\left(\frac{\mu_{k 3}-\ln x-\ln \bar{R}_{k 1}}{\varsigma}\right) \\
& \quad \times \exp \left[-\frac{\left(\ln x+\ln \bar{R}_{k 1}-\mu_{k 1}\right)^{2}}{2 \varsigma^{2}}\right]\left\{\prod_{\substack{j=1 \\
j \neq k}}^{K} \int_{0}^{x} \frac{\beta_{j 1}}{\delta_{j 1}}\right. \\
& \quad \times Q\left(\frac{\mu_{j 2}-\ln \delta_{j 1}-\ln \bar{R}_{j 1}}{\varsigma}\right) Q\left(\frac{\mu_{j 3}-\ln \delta_{j 1}-\ln \bar{R}_{j 1}}{\varsigma}\right) \\
& \left.\quad \times \exp \left[-\frac{\left(\ln \delta_{j 1}+\ln \bar{R}_{j 1}-\mu_{j 1}\right)^{2}}{2 \varsigma^{2}}\right] d \delta_{j 1}\right\} \frac{\beta_{k 1}}{x} d x .
\end{aligned}
$$

the fairness metric $F_{S P F}^{\prime}$ for the simplified PF scheme can be evaluated as

$$
F_{S P F}^{\prime}=\left[K \sum_{k=1}^{K}\left(S_{k 1}^{S P F}\right)^{2}\right]^{-1}
$$

In terms of the average data rate, the fairness metric $F_{S P F}$ is expressed by (35) shown at the top of next page. We note that in the simplified PF scheme, the value of $\delta_{k 1}$ is monitored by the average data rate $\bar{R}_{k 1}$. Thus, we expect that $F_{S P F}^{\prime}$ and $F_{S P F}$ are larger than $F_{C / I}^{\prime}$ and $F_{C / I}$, respectively. Intuitively, this is due to the fact that the user with poorer channel condition will get the relative $C / I$ increased by the lower $\bar{R}_{k 1}$. This will assure fairness in terms of both $F_{S P F}^{\prime}$ and $F_{S P F}$. Also note that the average data rate $\bar{R}_{k 1}$ in (31) only represents the initial monitoring constant. Once the throughput $\Lambda_{S P F}^{k}$ for the $k$ user is obtained by (32), $\bar{R}_{k 1}$ can be updated recursively using the algorithm proposed in [4], i.e., at time slot $t$, we have

$$
\bar{R}_{k 1}(t+1)=\left(1-1 / t_{c}\right) \bar{R}_{k 1}(t)+\Lambda_{S P F}^{k} / t_{c}
$$

where $t_{c}$ is a time window and $\Lambda_{S P F}^{k}$ is the current throughput for user $k$.

Next, we consider the throughput and fairness analysis for the conventional PF scheme in which the throughput monitoring is based on the user's relative data rate, $\zeta_{k 1}$, which is defined by

$$
\zeta_{k 1}=\frac{R_{k 1}}{\bar{R}_{k 1}}=\frac{W}{\bar{R}_{k 1}} \log _{2}\left(1+\alpha \gamma_{k 1}\right)
$$

where $\bar{R}_{k 1}$ is given by (30). Since the logarithm is a monotonic function, scheduling based on $\zeta_{k 1}$ given by (36) is akin to that based on $\delta_{k 1}$ given by (29) - both assure fairness. Since $\left\{\gamma_{k 1}\right\}_{k=1}^{K}$ are independent, the random variables $\left\{\zeta_{k 1}\right\}_{k=1}^{K}$ in (36) are also independent. Conditioning on $\zeta_{k 1}$, the system access probability for user $k$ is the product of the independent probabilities that each $\zeta_{j 1}<\zeta_{k 1}$ for $j \neq k$, namely
$\operatorname{Pr}\left[\max _{j \neq k}\left\{\zeta_{j 1}\right\} \leq \zeta_{k 1}\right]$, or equivalently,

$$
\begin{gathered}
\Phi\left(\gamma_{k 1}\right)=\operatorname{Pr}\left[\gamma_{11} \leq \frac{\left(1+\alpha \gamma_{k 1}\right)^{\bar{R}_{11} / \bar{R}_{k 1}-1}}{\alpha}, \ldots,\right. \\
\gamma_{(k-1) 1} \leq \frac{\left(1+\alpha \gamma_{k 1}\right)^{\bar{R}_{(k-1) 1} / \bar{R}_{k 1}-1}}{\alpha}, \\
\gamma_{(k+1) 1} \leq \frac{\left(1+\alpha \gamma_{k 1}\right)^{\bar{R}_{(k+1) 1} / \bar{R}_{k 1}}-1}{\alpha}, \ldots, \\
\left.\gamma_{K 1} \leq \frac{\left(1+\alpha \gamma_{k 1}\right)^{\bar{R}_{K 1} / \bar{R}_{k 1}-1}}{\alpha}\right],
\end{gathered}
$$

which yields the solution as

$$
\begin{array}{r}
\Phi\left(\gamma_{k 1}\right)=\prod_{\substack{j=1 \\
j \neq k}}^{K} \int_{0}^{\frac{\left(1+\alpha \gamma_{k 1}\right)^{\bar{R}_{j 1} / \bar{R}_{k 1}-1}}{\alpha}} \frac{\beta_{j 1}}{\gamma_{j 1}} Q\left(\frac{\mu_{j 2}-\ln \gamma_{j 1}}{\varsigma}\right) \\
\times Q\left(\frac{\mu_{j 3}-\ln \gamma_{j 1}}{\varsigma}\right) \exp \left[-\frac{\left(\ln \gamma_{j 1}-\mu_{j 1}\right)^{2}}{2 \varsigma^{2}}\right] d \gamma_{j 1} .
\end{array}
$$

Hence, the throughput for the conventional PF scheme is evaluated as

$\Lambda_{P F}=\sum_{k=1}^{K} \int_{0}^{\infty} \frac{W \beta_{k 1} \log _{2}\left(1+\alpha \gamma_{k 1}\right)}{\gamma_{k 1}} Q\left(\frac{\mu_{k 2}-\ln \gamma_{k 1}}{\varsigma}\right)$

$\times Q\left(\frac{\mu_{k 3}-\ln \gamma_{k 1}}{\varsigma}\right) \Phi\left(\gamma_{k 1}\right) \exp \left[-\frac{\left(\ln \gamma_{k 1}-\mu_{k 1}\right)^{2}}{2 \varsigma^{2}}\right] d \gamma_{k 1}$

The system access probability of user $k$ is given by

$$
\begin{aligned}
S_{k 1}^{P F}= & \int_{0}^{\infty} \Phi\left(\gamma_{k 1}\right) f_{c}\left(\gamma_{k 1}\right) d \gamma_{k 1} \\
= & \int_{0}^{\infty} \frac{\beta_{k 1}}{\gamma_{k 1}} Q\left(\frac{\mu_{k 2}-\ln \gamma_{k 1}}{\varsigma}\right) Q\left(\frac{\mu_{k 3}-\ln \gamma_{k 1}}{\varsigma}\right) \\
& \times \Phi\left(\gamma_{k 1}\right) \exp \left[-\frac{\left(\ln \gamma_{k 1}-\mu_{k 1}\right)^{2}}{2 \varsigma^{2}}\right] d \gamma_{k 1} .
\end{aligned}
$$

Thus, the fairness metric $F_{P F}^{\prime}$ for the conventional PF scheme is obtained as

$$
F_{P F}^{\prime}=\left[K \sum_{k=1}^{K}\left(S_{k 1}^{P F}\right)^{2}\right]^{-1}
$$

In terms of the average data rate, the fairness metric $F_{P F}$ is expressed by

$$
F_{P F}=\frac{\Lambda_{P F}^{2} / K}{\sum_{k=1}^{K}\left[\int_{0}^{\infty} W \log _{2}\left(1+\alpha \gamma_{k 1}\right) \Phi\left(\gamma_{k 1}\right) f_{c}\left(\gamma_{k 1}\right) d \gamma_{k 1}\right]^{2}}
$$

\section{Ensemble average throughput}

In Sections V.A - C, we have derived the throughput expressions of four scheduling schemes for the serving BS 1 , assuming a specific partitioning group $G_{1}$ with probability assignment $P$ given by (15). Since there are $2^{M}$ distinct ways of assigning the $M$ users to $G_{1}$, the ensemble average throughput for $\mathrm{BS} 1$ will be the sum of each throughput weighted by the corresponding probability of assignment. Let $\Lambda_{l}$ denote the throughput for the $l$ th assignment due to the RR scheme given by (23), or the maximum $C / I$ scheme given 


$$
F_{S P F}=\frac{\Lambda_{S P F}^{2} / K}{\sum_{k=1}^{K}\left\{\int_{0}^{\infty} W \log _{2}\left(1+\alpha \bar{R}_{k 1} \delta_{k 1}\right) \operatorname{Pr}\left[\max _{j \neq k}\left\{\delta_{j 1}\right\} \leq \delta_{k 1}\right] f_{c}\left(\delta_{k 1}\right) d \delta_{k 1}\right\}^{2}} .
$$

by (25), or the simplified PF scheme given by (32), or the conventional PF scheme given by (39). Then, the ensemble average throughput for BS 1 can be expressed by

$$
\Lambda_{a v}=\sum_{l=1}^{2^{M}} \Lambda_{l} P_{l}
$$

where $P_{l}$ is the corresponding probability assignment and $\left\{P_{l}\right\}$ is subject to the constraint given by (14). Clearly, in the case that no user is served by BS 1 (e.g., $l=1$ in Table 1 ), we have $\Lambda_{l}=0$, and in the case that only one user is served by BS 1 (e.g., $l=2,3$, or 4 in Table 1 ), the throughputs $\Lambda_{l}$ are equal for all four scheduling schemes.

\section{E. Peak data throughput}

The result (43) gives the ensemble average data rate for BS 1 , which is also applicable to BS 2 and BS 3 by symmetry. Thus, it provides the average data throughput in the active region shown in Fig. 1. For comparison, in this subsection we will also derive the peak data throughput in the active region. The peak data throughput is defined as the highest data rate that can be supported at any instant. From (11), we see that this can be achieved by the maximum received $C / I$, and hence the peak data throughput, denoted by $R_{\max }$, is formulated as

$$
R_{\max }=\int_{0}^{\infty} W \log _{2}\left(1+\alpha \gamma_{\max }\right) f\left(\gamma_{\max }\right) d \gamma_{\max }
$$

where $\gamma_{\max }$ represents the maximum received $C / I$ in the active region. Note that, the definition of the peak data throughput in (44) follows the idea of estimating the channel capacity in Rayleigh fading environment presented in [31], which is not an absolute maximum value, but calculated in an average sense. This is because the $\gamma_{\max }$ is a random variable that varies in time.

For a fixed user $k$, the maximum received $C / I$ from the BS set $S$ due to FCS, denoted by $\gamma_{\text {max }}^{k}$, is given by $\gamma_{\max }^{k}=\max \left\{\gamma_{k 1}, \gamma_{k 2}, \gamma_{k 3}\right\} ; \gamma_{\max }$ will then be the largest random variable among $\left\{\gamma_{\max }^{k}\right\}_{k=1}^{M}$ of all users, namely, $\gamma_{\max }=\max _{k}\left\{\gamma_{\max }^{k}\right\}$, or equivalently,

$$
\gamma_{\text {max }}=\max _{k, i}\left\{\gamma_{k i}\right\} \quad k=1,2, \cdots, M ; \quad i=1,2,3 .
$$

The CDF of $\gamma_{\max }$ is the probability that the $\gamma_{k i}$ in all $3 M$ quantities are simultaneously less than or equal to some specified value. In view of (7), the CDF of $\gamma_{\max }$ is shown to be

$$
\Gamma\left(\gamma_{\max }\right)=\prod_{k=1}^{M} \prod_{i=1}^{3} Q\left(\frac{\mu_{k i}-\ln \gamma_{\max }}{\varsigma}\right) .
$$

This is the distribution of the maximum received $C / I$ in the active region, selected from $3 M$ quantities of three BSs and

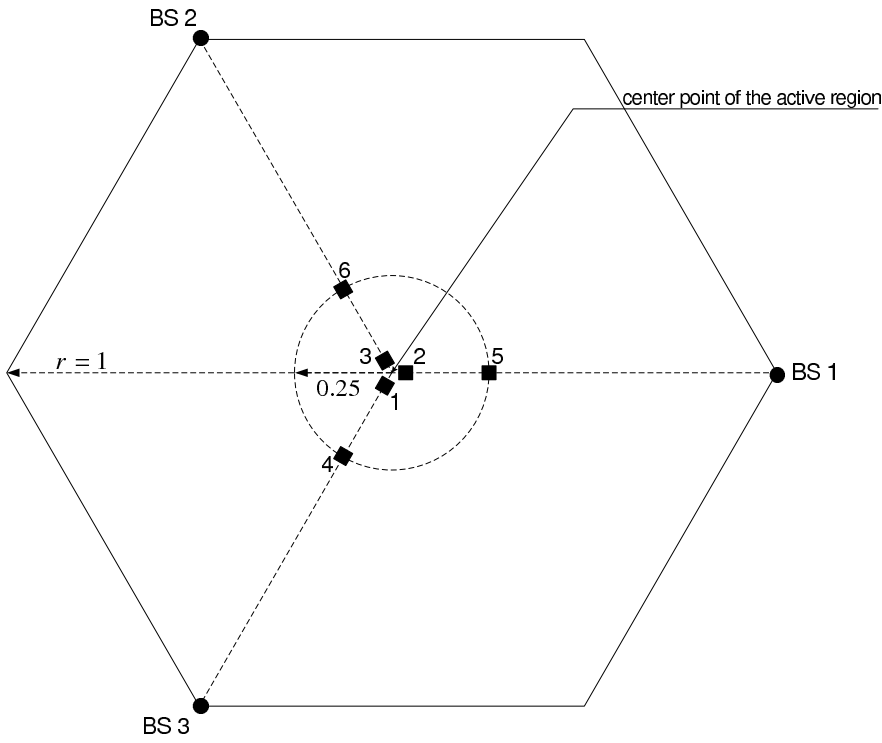

Fig. 4. The active region for FCS and the user distribution scenarios $1-6$ for numerical examples.

$M$ users. The corresponding PDF for $\gamma_{\max }$ is obtained as

$$
f\left(\gamma_{\max }\right)=\frac{d}{d \gamma_{\max }}\left[\prod_{k=1}^{M} \prod_{i=1}^{3} Q\left(\frac{\mu_{k i}-\ln \gamma_{\max }}{\varsigma}\right)\right] .
$$

Substituting (47) into (44) yields the peak data throughput

$$
\begin{aligned}
& R_{\max }=\int_{0}^{\infty} W \log _{2}\left(1+\alpha \gamma_{\max }\right) \\
& \times d\left[\prod_{k=1}^{M} \prod_{i=1}^{3} Q\left(\frac{\mu_{k i}-\ln \gamma_{\max }}{\varsigma}\right)\right] .
\end{aligned}
$$

Note that in computing $R_{\max }$, we should first evaluate $d\left[\prod_{k=1}^{M} \prod_{i=1}^{3} Q\left(\frac{\mu_{k i}-\ln \gamma_{\max }}{\varsigma}\right)\right]$, then (48) is calculated numerically. The partial integration rule $\int_{0}^{\infty} f(x) d g(x)=$ $\lim _{x \rightarrow \infty} f(x) g(x)-\lim _{x \rightarrow 0} f(x) g(x)-\int_{0}^{\infty} g(x) d f(x)$ is not applicable to (48). The reason is that $\lim _{x \rightarrow \infty} f(x) g(x) \rightarrow \infty$.

\section{NUMERICAL/SIMULATION EXAMPLES AND DISCUSSIONS}

This section presents some numerical/simulation examples to illustrate the behavior of throughput and fairness as a function of various scheduling schemes with/without FCS and system parameters.

Fig. 4 shows the active region and six possible user locations. The cell distance $r$ is normalized to 1 . To facilitate studying FCS, the distance between the center of the active region and users $4-6$ is 0.25 . Users $1-3$ are assumed to be very close to the center so that the distance can be approximated to be zero. In actual numerical evaluation we assume that users 13 locate at the center of the active region with coordinate $[0,0]$. 


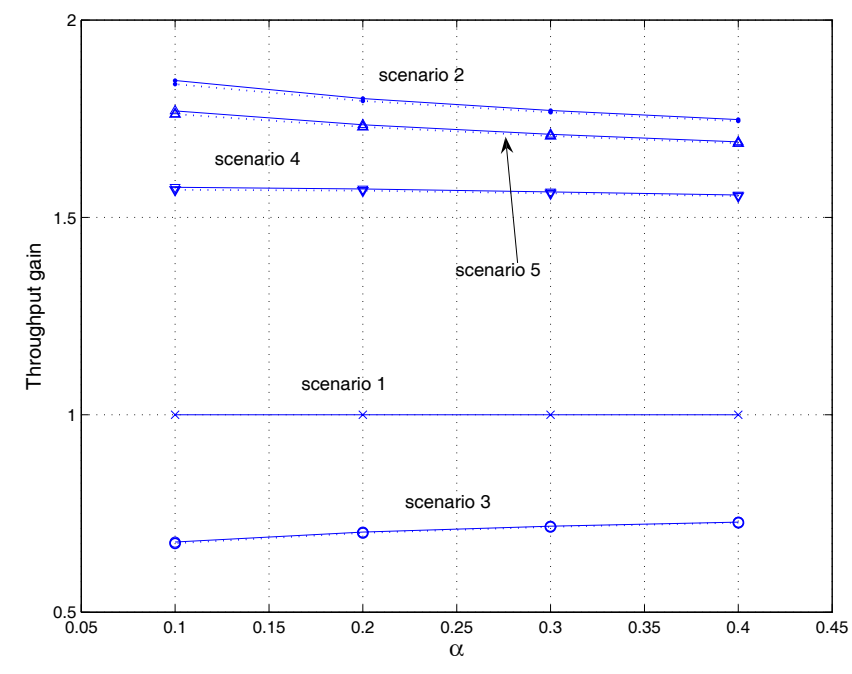

Fig. 5. Throughput gain versus $\alpha$ with Round Robin scheme.

We further assume that the bandwidth $W$, average envelope power $\eta^{2}$, and standard deviation $\sigma(\mathrm{dB})$ are set to $3.84 \mathrm{MHz}$, 1 and 8, respectively. Clearly, without FCS, only users 1, 2, 3 and 5 can connect to BS 1 . With FCS, there are $2^{6}=64$ distinct ways of assigning six users to $G_{1}$ to be served by BS 1. We consider five scenarios in which only two users are connected to BS 1, namely, senario 1: users 2 and 5 without FCS; scenario 2: users 2 and 5 with FCS; scenario 3: users 1 and 2 without FCS; scenario 4: users 1 and 2 with FCS; and scenario 5: users 4 and 5 with FCS. This is sufficient to illustrate our discussions.

Fig. 5 plots the normalized throughput gains for the RR scheme against the parameter $\alpha$. Specifically, we first compute the throughputs of the abovementioned five scenarios with RR scheme. The throughput gains are then evaluated by normalizing each throughput with that of scenario 1 . Recall from (10) that the parameter $\alpha$ is equivalent to $\left(E_{b} / I_{0}\right)_{k}$, which is required to achieve a certain PER performance. In our numerical evaluation the value of $\alpha$ is assumed to be 0.1 , $0.2,0.3$ and 0.4 , which corresponds to $\left(E_{b} / I_{0}\right)_{k}=8.4 \mathrm{~dB}, 5.4$ $\mathrm{dB}, 3.6 \mathrm{~dB}$ and $2.5 \mathrm{~dB}$, respectively. It can be seen that FCS can substantially improve the throughput performance. For example, for $\alpha=0.1$, the throughput gain of scenario 2 (users 2 and 5 with FCS) over scenario 1 (users 2 and 5 without FCS) is 1.85 . Without FCS, the throughput of scenario 3 (users 1 and 2 without FCS) is below the baseline (scenario 1). The gain is approximately 0.7 . With FCS this gain is increased approximately to 1.6 in scenario 4 (users 1 and 2 with FCS). The improvement is up to about $1.6 / 0.7=2.3$. In order to validate the analysis, computer simulation results have also been included in Fig. 5 and the subsequent Figs. 6 - 9. The simulation results are shown as the dotted lines in the figure. From the plots in Figs. 5 - 9 we see that the simulation results confirm the feasibility of the theoretical analysis.

Figs. 6, 7 and 8 show the throughput comparisons of RR, maximum $C / I$, simplified PF and conventional PF with FCS for scenario 2, scenario 5 and scenario 4, respectively. Besides the throughput of BS 1 , the average data rate of each user is also given. For example, for scenario 2 in Fig. 6, curves

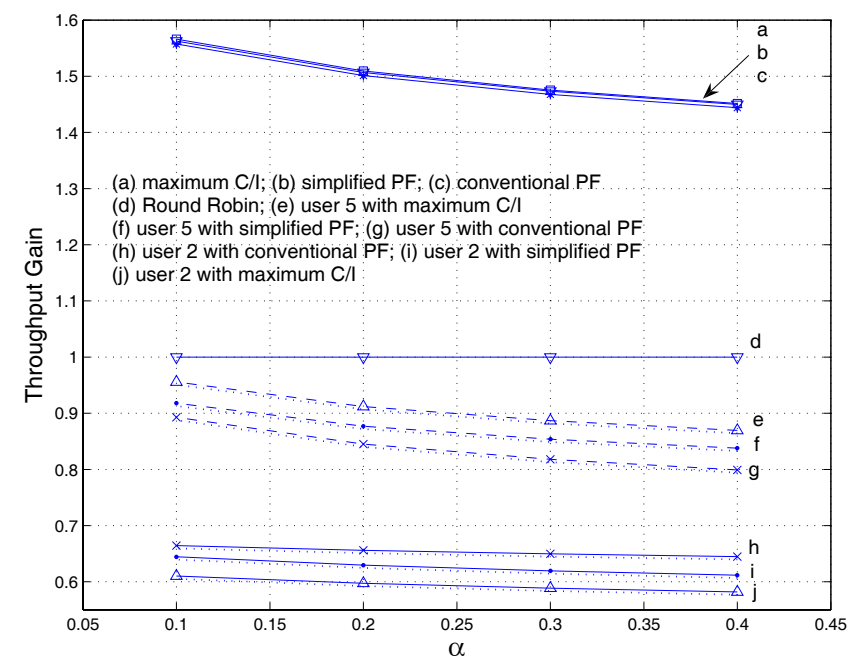

Fig. 6. Throughput performance comparison for scenario 2 .

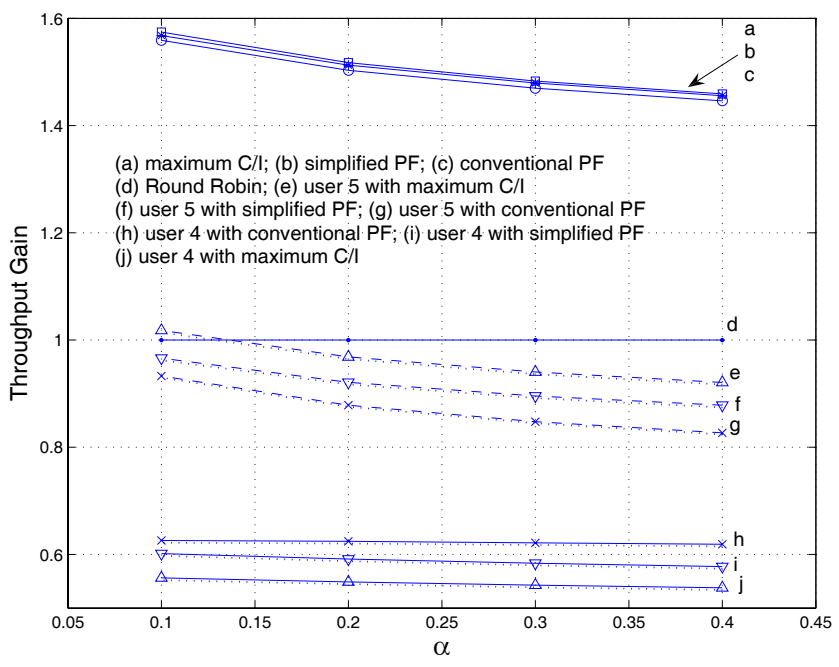

Fig. 7. Throughput performance comparison for scenario 5.

a-d represent the throughput gain of BS 1, while curves e-j represent the average data rate gain of users 2 and 5. Without loss of generality, in Figs. 6 - 8, we have chosen the throughput of BS 1 with RR scheme as a baseline.

From these three figures, several conclusions can be drawn. First, the RR scheme gives the worst performance of the four schemes examined in this paper. This is expected since the RR scheme does not take into account the channel conditions among the users. Hence, although FCS can help prevent the users with deep fade due to small-scale Rayleigh fading and large-scale lognormal fading from connecting to BS 1 , the throughput descreases when the fixed time slot allocation in the RR scheme is made to the user with small $C / I$. Second, the throughput performance depends on the users' location. In Fig. 8, the maximum $C / I$, simplified PF and conventional $\mathrm{PF}$ schemes have the same throughput gain for scenario 4 , while in Figs. 6 and 7 for scenario 2 and scenario 5, the maximum $C / I$ scheme provides the best performance and the simplified PF scheme outperforms the conventional PF scheme. Moreover, we see that as the users' location difference increases, the performance gap between the individual users 


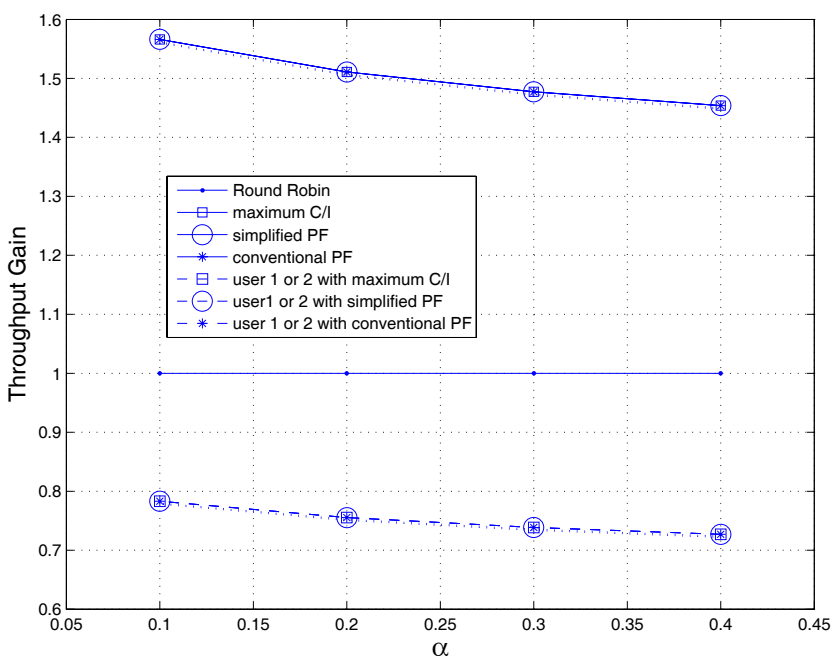

Fig. 8. Throughput performance comparison for scenario 4 .

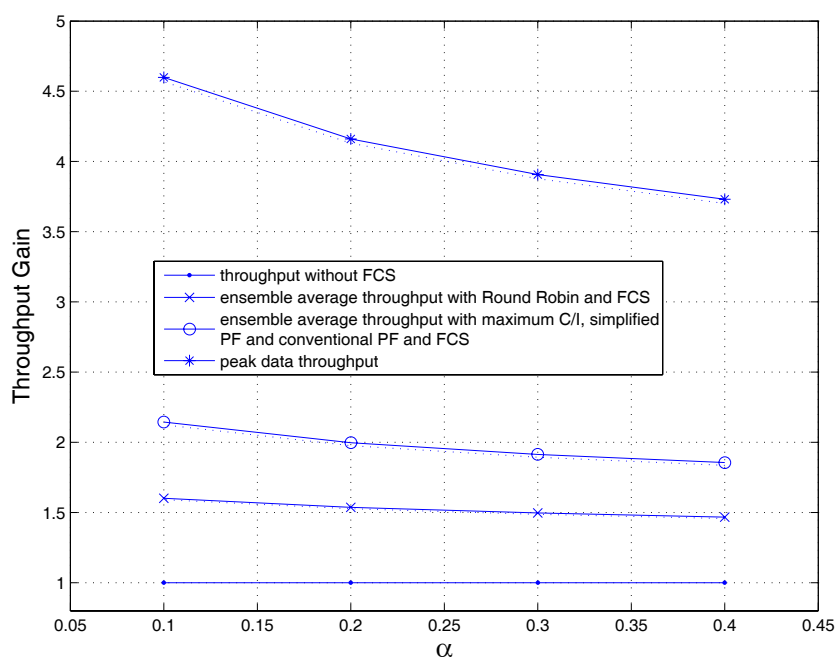

Fig. 9. Comparison of the ensemble average throughput and the peak data throughput for scenario 6 .

is increasingly higher. For instance, for the maximum $C / I$ scheme, the gain ratio of the average data rate between users 1 and 2 in Fig. 8 for scenario 4 is 1, 1, 1 and 1 for $\alpha=0.1,0.2$, 0.3 and 0.4 , respectively. It becomes $1.5987,1.5618,1.5432$ and 1.5313 between users 5 and 2 in Fig. 6 for scenario 2, and $1.8299,1.7652,1.7326$ and 1.7118 between users 5 and 4 in Fig. 7 for scenario 5. This implies that an increase of location difference will degrade the fairness between the users when the fairness metric is evaluated in terms of the average data rate. Third, the conventional PF scheme can achieve a better trade-off between the fairness in terms of the average data rate and throughput than the simplified PF scheme. In Figs. 6 and 7, the throughput gain of the conventional PF scheme (curve c) is only slightly inferior to that of the simplified PF scheme (curve b), but the average data rate gap of the two users in the conventional PF scheme (curves $g$ and $h$ ) is considerably smaller than that in the simplified PF scheme (curves $f$ and i). This indicates that without incurring much throughput performance sacrifice, in contrast to the simplified PF scheme, the conventional PF scheme will have a better balance on the average data rate bewteen the users, which can lead to the fairness improvement. Finally, due to the highly non-linear feature of the logarithmic relationship given by (11), the throughput gains are sensitive to the parameter $\alpha$.

Fig. 9 shows the performance comparison of ensemble average throughput with/without FCS and peak data throughput in the active region. For simplicity, we assume that there are only three users in the active region locating at positions 1,2 and 3, as shown in Fig. 4. This user distribution is referred to here as scenario 6 . The ensemble average throughput without FCS serves as the baseline. With FCS, the average throughput is computed using (43). The probability assignments $P_{l}$ are computed using (15) and listed in the last column of Table 1. Since we assume that the coordinate for all three users is $[0,0]$, the mean $\mu_{k i}$ would be the same for all $k=1,2,3$ and $i=1,2,3$. Then, the quantity $\prod_{k=1}^{M} \prod_{i=1}^{3} Q\left(\frac{\mu_{k i}-\ln \gamma_{\max }}{\varsigma}\right)$ in (48) can be simplified to

$$
\prod_{k=1}^{M} \prod_{i=1}^{3} Q\left(\frac{\mu_{k i}-\ln \gamma_{\max }}{\varsigma}\right)=\left[Q\left(\frac{\mu-\ln \gamma_{\max }}{\varsigma}\right)\right]^{9}
$$

where $\mu_{k i}=\mu$ for all $k, i$. Substituting (49) into (48), we obtain the peak data throughput

$$
\begin{aligned}
R_{\max }= & \frac{9 W}{\sqrt{2 \pi \varsigma^{2}}} \int_{0}^{\infty} \frac{\log _{2}(1+\alpha x)}{x}\left[Q\left(\frac{\mu-\ln x}{\varsigma}\right)\right]^{8} \\
& \times \exp \left[-\frac{(\ln x-\mu)^{2}}{2 \varsigma^{2}}\right] d x .
\end{aligned}
$$

The results in Fig. 9 indicate that with techniques of AMC, FCS and downlink scheduling 3G WCDMA networks can provide very high peak data rate to support high-traffic load. This is important to such applications as web browsing, streaming audio/video and file transfer involving large files being sent to the users in the internet era of the downlink WCDMA system.

Table 2 shows the fairness comparison for the four different scheduling schemes in scenarios 2, 4 and 5. The parameter $\alpha$ is set to 0.4 . The metric values state that the maximum $C / I$ scheme has the worst fairness performance as compared to the other schemes in terms of both system access probability and average data rate. In terms of the system access probability, the RR scheme can give a totally fair allocation regardless of users' locations and channel conditions. However, in terms of the average data rate, the RR scheme becomes increasingly unfair as the difference of users' channel conditions increases. By comparing $F_{P F}$ with $F_{S P F}$ and $F_{P F}^{\prime}$ with $F_{S P F}^{\prime}$, we note that in general the conventional PF scheme can provide better fairness performance than the simplified PF scheme in terms of both metric criteria.

\section{REFERENCES}

[1] H. Holman and A. Toskala, WCDMA for UMTS: Radio Access for Thrid Generation Mobile Communication. Hoboken, NJ: John Wiley \& Sons, 3rd Edition, 2004.

[2] S. Parkvall, E. Dahlman, P. Frenger, P. Beming, and M. Persson, "The evolution of WCDMA towards higher speed downlink packet data access," in Proc. IEEE VTC Spring 2001, vol. 3, pp. 2287-2291, Rhodes, Greece, May 2001.

[3] P. Bender, P. Black, M. Grob, R. Padovani, N. Sindhushayana, and A. Viterbi, "CDMA/HDR: a bandwidth-efficient high-speed wireless data service for nomadic users," IEEE Commun. Mag., vol. 38, pp. 70-77, July 2000. 
TABLE II

FAIRNESS COMPARISON $(\alpha=0.4)$

\begin{tabular}{|c|c|c|c|c|}
\hline Scheduling & Fairness metric & scenario 2 & scenario 4 & scenario 5 \\
\hline \multirow{3}{*}{ Round Robin } & $F_{R R}$ & 0.9882 & 1.0000 & 0.9791 \\
\cline { 2 - 5 } & $F_{R R}^{\prime}$ & 1.0000 & 1.0000 & 1.0000 \\
\hline \multirow{3}{*}{ Maximum $C / I$} & $F_{C / I}$ & 0.9623 & 1.0000 & 0.9355 \\
\cline { 2 - 5 } & $F_{C / I}^{\prime}$ & 0.9797 & 1.0000 & 0.9656 \\
\hline \multirow{3}{*}{ Simplified PF } & $F_{S P F}$ & 0.9762 & 1.0000 & 0.9590 \\
\cline { 2 - 5 } Conventional PF & $F_{S P F}^{\prime}$ & 0.9948 & 1.0000 & 0.9915 \\
\cline { 2 - 5 } & $F_{P F}$ & 0.9887 & 1.0000 & 0.9798 \\
\hline
\end{tabular}

[4] A. Jalali, R. Padovani, and R. Pankaj, "Data throughput of CDMAHDR a high efficiency-high data rate personal communication wireless system," in Proc. IEEE VTC Spring 2000, vol. 3, pp. 1854-1858, Tokyo, May 2000.

[5] 3GPP Technical Specification, "Physical layer aspects of UTRA high speed downlink packet access," 3GPP TR 25.848 v4.0.0, Mar. 2001.

[6] Qualcomm, Inc., 1xEV: 1xEVolution IS-856 TIA/EIA Standard, Airlink Overview, Nov. 2001.

[7] Qualcomm, Inc., "HSDPA for improved downlink data transfer," Qualcomm CDMA Technologies White Paper, Oct. 2004.

[8] S. Benedetto et al, "MHOMS: high-speed ACM modem for satellite applications," IEEE Wireless Commun. Mag., vol. 12, pp. 66-77, Apr. 2005.

[9] J. Yang, A. K. Khandani and N. Tin, "Statistical decision making in adaptive modulation and coding for $3 \mathrm{G}$ wireless systems," IEEE Trans. Veh. Technol., vol. 54, pp. 2066-2073, Nov. 2005.

[10] H. Furukawa, K. Hamabe, and A. Ushirokawa, "SSDT - site selection diversity transmission power control for CDMA forward link," IEEE J. Select. Areas Commun., vol. 18, pp. 1546-1554, Aug. 2000.

[11] A. Das et al, "Network controlled cell selection for the high speed downlink packet access in UMTS," in Proc. IEEE WCNC 2004, vol. 4, pp. 1975-1979, Mar. 2004.

[12] A. Sang, X. D. Wang, M. Madihian, and R. D. Gitlin, "A flexible downlink scheduling scheme in cellular packet data systems," IEEE Trans. Wireless Commun., vol. 5, pp. 568-577, Mar. 2006.

[13] E. Lim and S. Kim, "Transmission rate scheduling with fairness constraints in downlink of CDMA data networks," IEEE Trans. Veh. Technol., vol. 54, pp. 328-337, Jan. 2005.

[14] R. Kwan and C. Leung, "Downlink scheduling optimization in CDMA networks," IEEE Commun. Lett., vol. 8, pp. 611-613, Oct. 2004.

[15] M. A. Haleem and R. Chandramouli, "Adaptive downlink scheduling and rate selection: a cross-layer design," IEEE J. Select. Areas Commun., vol. 23, pp. 1287-1297, June 2005.

[16] S. Y. Kim and H. M. Baek, "On the economic aspects of downlink scheduling in DS-CDMA systems: base-station density perspective," IEEE Trans. Veh. Technol., vol. 55, pp. 1594-1602, Sept. 2006.

[17] A. J. Viterbi, CDMA: Principles of Spread Spectrum Communications. Addison Wesley, 1995.

[18] 3GPP Technical Specification, "Stage 2 functional specification of user equipment (UE) positioning in UTRAN (Release 7)," 3GPP TS 25.305 version 7.3.0, June 2006.

[19] 3GPP Technical Specification, "Location services (LCS), service description, stage 1," 3G TS 22.071 version 7.4.0, Dec. 2005.

[20] K. S. Gilhousen, I. M. Jacobs, R. Padovani, A. J. Viterbi, L. A. Weaver, and C. E. Wheatley, "On the capacity of a cellular CDMA system," IEEE Trans. Veh. Technol., vol. 40, pp. 303-312, May 1991.

[21] G. L. Stuber, Principles of Mobile Communication. Boston: Kluwer Academic, 2nd Edition, 2001.

[22] F. Berggren and R. Jantti, "Asymptotically fair transmission scheduling over fading channels," IEEE Trans. Wireless Commun., vol. 3, pp. 326336, Jan. 2004

[23] J. M. Holtzman, "Asymptotic analysis of proportional fair algorithm," in Proc. IEEE PIMRC 2001, vol. 2, pp. F33-F37, San Diego, Sept. 2001.

[24] H. Fu and D. I. Kim, "Analysis of throughput and fairness with downlink scheduling in WCDMA networks," IEEE Trans. Wireless Commun., vol. 5, pp. 2164-2174, Aug. 2006.

[25] J. G. Proakis, Digital Communications, 4th ed. New York: McGraw-Hill, 2001.

[26] M. Fisz, Probability Theory and Mathematical Statistics. New York: Wiley, 3rd Edition, 1963.
[27] J. M. Wozencraft and I. M. Jacobs, Principles of Communication Engineering. Waveland Press, 1990.

[28] D. M. Chiu and R. Jain "Analysis of the increase and decrease algorithm for congestion avoidance in computer networks," Computer Networks and ISDN System, vol. 17, pp. 1-14, June 1989.

[29] D. I. Kim, E. H. Hossain, and V. K Bhargava, "Downlink joint rate and power allocation in cellular multirate WCDMA systems," IEEE Trans. Wireless Commun., vol. 2, pp. 69-80, Jan. 2003.

[30] A. Papoulis, Probability, Random Variables, and Stochastic Processes. New York: McGraw-Hill, 3rd Edition, 1991.

[31] W. C. Y. Lee, "Estimate of channel capacity in Rayleigh fading environment," IEEE Trans. Veh. Technol., vol. 39, pp. 187-189, Aug. 1990.

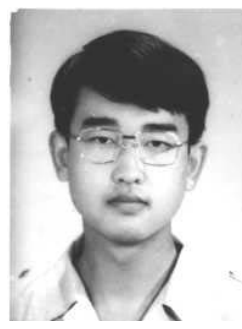

Hua Fu was with the School of Engineering and Electronics, University of Edinburgh, U.K. from 2001 to 2003. From April, 2003 to January, 2004, he was with the School of Engineering Science, Simon Fraser University, Canada. Since January, 2004, he has been with the Department of Electrical and Computer Engineering, National University of Singapore. He was a Visiting Fellow at Simon Fraser University in August, 2007. His has conducted research in the areas of digital communication over fading channels, multiuser detection, digital signal processing, and WCDMA mobile networks. He made contributions on receiver design and performance analysis of MDPSK signaling over diversity, non-identically distributed, Rayleigh channels with symmetric/asymmetric fading spectrum and downlink scheduling analysis of 3GPP-WCDMAHSDPA/3GPP2-CDMA-HDR mobile networks.

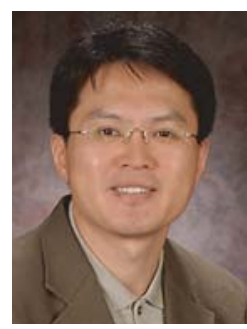

Dong In Kim (S'89-M'91-SM'02) received the B.S. and M.S. degrees in Electronics Engineering from Seoul National University, Seoul, Korea, in 1980 and 1984, respectively, and the M.S. and Ph.D. degrees in Electrical Engineering from the University of Southern California (USC), Los Angeles, in 1987 and 1990, respectively.

From 1984 to 1985 , he was a Researcher with Korea Telecom Research Center, Seoul. From 1986 to 1988 , he was a Korean Government Graduate Fellow in the Department of Electrical Engineering, USC. From 1991 to 2002, he was with the University of Seoul, Seoul, leading the Wireless Communications Research Group. From 2002 to 2007, he was a tenured Full Professor in the School of Engineering Science, Simon Fraser University, Burnaby, BC, Canada. From 1999 to 2000, he was a Visiting Professor at the University of Victoria, Victoria, BC. Since 2007, he has been with Sungkyunkwan University (SKKU), Suwon, Korea, where he is a Professor and SKKU Fellow in the School of Information and Communication Engineering. Since 1988, he is engaged in the research activities in the areas of cellular radio networks and spread-spectrum systems. His current research interests include wideband, broadband, and ultrawideband (UWB) communications for high-data-rate wireless multimedia, cooperative and cognitive radio (CR) techniques, and cross-layer design for CR systems.

Dr. Kim was an Editor for the IEEE Journal on Selected Areas in Communications: Wireless Communications Series and also a Division Editor for the Journal of Communications and Networks. He is currently an Editor for Spread Spectrum Transmission and Access for the IEEE Transactions on Communications and an Editor for the IEEE Transactions on Wireless Communications. 